\title{
EXPLICIT EXPRESSIONS FOR STURM-LIOUVILLE OPERATOR PROBLEMS
}

\author{
by LUCAS JODAR \\ (Received 6th January 1986)
}

\section{Introduction}

Throughout this paper $H$ will denote a complex separable Hilbert space and $L(H)$ denotes the algebra of all bounded linear operators on $H$. If $T$ lies in $L(H)$, its spectrum $\sigma(T)$ is the set of all complex numbers $z$ such $z I-T$ is not invertible in $L(H)$ and its compression spectrum $\sigma_{\text {comp }}(T)$ is the set of all complex numbers $z$ such that the range $(z I-T)(H)$ is not dense in $H([3$, p. 240]). This paper is concerned with the SturmLiouville operator problem

$$
\begin{gathered}
X^{(2)}(t)-\lambda Q X(t)=0 \\
E_{1} X(0)+E_{2} X^{(1)}(0)=0 \\
F_{1} X(a)+F_{2} X^{(1)}(a)=0, \quad 0 \leqq t \leqq a
\end{gathered}
$$

where $\lambda$ is a complex parameter and $X(t), Q, E_{i}, F_{i}$, for $i=1,2$, and $t \in[0, a]$, are bounded operators in $L(H)$. For the scalar case, the classical Sturm-Liouville theory yields a complete solution of the problem, see [4], and [7]. For the finite-dimensional case, second order operator differential equations are important in the theory of damped oscillatory systems and vibrational systems ([2, 6]). Infinite-dimensional differential equations occur frequently in the theory of stochastic processes, the degradation of polymers, infinite ladder network theory in engineering [1, 17], denumerable Markov chains, and moment problems $[10,20]$. Sturm-Liouville operator problems have been studied by several authors and with several techniques $([12,13,14,15,16])$.

In order to solve the operator differential equation

$$
X^{(2)}(t)-\lambda Q X(t)=0
$$

and in an analogous way to the scalar case, we obtain a fundamental set of solutions for the equation (2.1), from the existence of solutions for the algebraic characteristic operator equation

$$
X^{2}-\lambda Q=0
$$


Thus, explicit expressions for any solution of (2.1) are given in terms of solutions of the algebraic equation (3.1). In this sense, this paper can be regarded as a continuation of [8] and [9]. Notice that for the operator case, the equation (3.1) can be unsolvable, for example, if $\lambda Q$ is an unilateral weighted shift operator, the equation (3.1) is unsolvable $([19$, p. 63]).

The resolution problem of the equation (3.1) is related to the problem of the existence of a linear factorization of the polynomial operator $L(z)=z^{2}-\lambda Q$. So, for the finitedimensional case, the equation (3.1) is solvable, if and only if, the companion operator

$$
C_{L}=\left[\begin{array}{rr}
0 & I \\
-Q & 0
\end{array}\right]
$$

is diagonable. The infinite-dimensional case is treated in [18], in a more general context. In order to obtain explicit solutions of the equation (3.1), the Riesz-Dunford functional calculus yields such an expression. In fact, if $\alpha$ is a real number with $0 \leqq \alpha<2 \pi, H_{\alpha}$ is the half-line $\{-r \exp (i \alpha), r \geqq 0\}$, and $D_{\alpha}$ is the complementary set of $H_{\alpha}$ in the complex plane, there is an analytic determination of the complex logarithm $\log _{\alpha}$, defined in $D_{\alpha}$. Thus, if the spectrum $\sigma(\lambda Q)$ is contained in $D_{\alpha}$, from the Riesz-Dunford functional calculus, [5], a solution of the equation (3.1) is given by the expression $X_{0}=$ $\exp \left(\log _{\alpha}(\lambda Q) / 2\right)$. Moreover, if the operator $\lambda Q$ has a finite spectrum, this operator $\mathrm{x}_{0}$ can be computed as a polynomial in $\lambda Q$, see [5, Th. VII. 16]. In particular, this computation is available for the finite-dimensional case. The above condition $\sigma(\lambda Q) \subset D_{\alpha}$ is satisfied by a lot of operators, as it is shown in the following example.

Example 1. Let $Q$ be an operator in $L(H)$ such that its spectrum $\sigma(Q)$ is contained in $\mathbb{C} \sim L$, where $L$ is a half-line, $L=\{-r \exp (i s) ; r \leqq 0\}$. If we write the complex number $\lambda$, in the form $\lambda=|\lambda| \exp (i t)$, for some $t$ in $[0,2 \pi[$, then one has $\sigma(\lambda Q)=\{\lambda z ; z \in \sigma(Q)\}$ and taking $\alpha=s+t$, it turns out that $\sigma(\lambda Q) \subset D_{\alpha}$, notice that if $s+t \geqq 2 \pi$, then $D_{\alpha}=D_{\alpha-2 \pi}$, and $\alpha^{\prime}=\alpha-2 \pi$, satisfies $\sigma(\lambda Q) \subset D_{\alpha^{\prime}}$. In particular, any operator $Q$ whose spectrum $\sigma(Q)$ is contained in a proper sector of the complex plane satisfies the above condition.

We study separately the cases $\lambda=0$ and $\lambda \neq 0$, and we suppose that for $\lambda \neq 0$, the spectrum $\sigma(\lambda Q)$ is contained in $D_{\alpha}$, for some $\alpha$ in $[0,2 \pi[$.

\section{Explicit solutions for Sturm-Liouville operator problems}

Let us consider the operator matrix

$$
\left[\begin{array}{ll}
A_{11} & A_{12} \\
A_{21} & A_{22}
\end{array}\right]: H \oplus H \rightarrow H \oplus H
$$

where $A_{i j}, 1 \leqq i, j \leqq 2$, are operators in $L(H)$. If $A_{22}$ is invertible, then it follows that

$$
A=\left[\begin{array}{cc}
I & A_{12} A_{22}^{-1} \\
0 & I
\end{array}\right]\left[\begin{array}{cc}
A_{11}-A_{12} A_{22}^{-1} A_{21} & 0 \\
0 & A_{22}
\end{array}\right]\left[\begin{array}{cc}
I & 0 \\
A_{22}^{-1} A_{21} & I
\end{array}\right] .
$$


Since the first and third factor in the right hand side of this identity are invertible in $L(H \oplus H)$, it follows that $A$ is invertible if and only if the operator $W=A_{11}-A_{12} A_{22}^{-1} A_{21}$ is invertible. In this case, it is easy to show that

$$
A^{-1}=\left[\begin{array}{cc}
W^{-1} & -W^{-1} A_{12} A_{22}^{-1} \\
-A_{22}^{-1} A_{21} W^{-1} & A_{22}^{-1} A_{21} W^{-1} A_{12} A_{22}^{-1}+A_{22}^{-1}
\end{array}\right] .
$$

Otherwise, if $A_{11}$ is invertible, one gets

$$
A=\left[\begin{array}{cc}
I & 0 \\
A_{22} A_{11}^{-1} & I
\end{array}\right]\left[\begin{array}{cc}
A_{11} & 0 \\
0 & A_{22}-A_{21} A_{2}^{-1} A_{12}
\end{array}\right]\left[\begin{array}{cc}
I & A_{11}^{-1} A_{12} \\
0 & I
\end{array}\right] .
$$

Thus, $A$ is invertible if and only if, $V=A_{22}-A_{21} A_{11}^{-1} A_{12}$ is invertible. In this case one easily computes that

$$
A^{-1}=\left[\begin{array}{cc}
A_{11}^{-1} A_{12} V^{-1} A_{21} A_{11}^{-1}+A_{11}^{-1} & -A_{11}^{-1} A_{12} V^{-1} \\
-V^{-1} A_{21} A_{11}^{-1} & V^{-1}
\end{array}\right] .
$$

From here the following result is proved.

Lemma 1. Let $A$ be the operator defined by (1.2). Then it follows that:

i) If $A_{22}$ is invertible, the operator $A$ is invertible if and only if, the operator $W=$ $A_{11}-A_{12} A_{22}^{-1} A_{21}$ is invertible. In this case $A^{-1}$ is given by (2.2).

(ii) If $A_{11}$ is invertible, the operator $A$ is invertible if and only if, the operator $V=$ $A_{22}-A_{21} A_{11}^{-1} A_{12}$ is invertible. In this case $A^{-1}$ is given by (3.2)

The following result yields an explicit expression for any solution of the operator differential equation (2.1) under the compatibility hypothesis of the algebraic equation (3.1).

Lemma 2. Let us consider the operator differential equation (2.1), where $\lambda \neq 0$ and $Q$ is an invertible operator such that $\sigma(\lambda Q) \subset D_{\alpha}$, for some $\alpha \in\left[0,2 \pi\left[\right.\right.$. Let $X_{0}=\exp \left(\left(\log _{\alpha}(\lambda Q) / 2\right)\right.$ be a square root of $\lambda Q$, then for any solution $X$ of (2.1), there are operators $C$ and $D$ such that

$$
X(t)=\exp \left(t X_{0}\right) C+\exp \left(-t X_{0}\right) D
$$

These operators $C$ and $D$ are uniquely determined by the expressions

$$
C=X(0) / 2+X_{0}^{-1} X^{(1)}(0) / 2 ; \quad D=X(0) / 2-X_{0}^{-1} X^{(1)}(0) / 2 .
$$

Proof. It is clear that $X$ given by (4.2) is a solution of the equation (2.1). From the uniqueness for a Cauchy problem, [11], it is sufficient to show that given $C_{0}=X(0)$ and $C_{1}=X^{(1)}(0)$, there are operators $C$ and $D$ in $L(H)$ which satisfy the condition (5.2). From 
(4.2), it is equivalent to prove the existence of operators $C$ and $D$ such that

$$
X(0)=C_{0}=C+D ; \quad X^{(1)}(0)=C_{1}=X_{0} C-X_{0} D
$$

that is,

$$
\left[\begin{array}{cc}
I & I \\
X_{0} & -X_{0}
\end{array}\right]\left[\begin{array}{l}
C \\
D
\end{array}\right]=\left[\begin{array}{l}
C_{0} \\
C_{1}
\end{array}\right]
$$

From the spectral mapping theorem, [5, p. 569], $X_{0}$ is an invertible operator and from Lemma 1, the first operator matrix in the left hand side of (6.2) is invertible in $L(H \oplus H)$. Thus, from (6.2) and Lemma 1, it follows that

$$
\left[\begin{array}{l}
C \\
D
\end{array}\right]=\left[\begin{array}{cc}
I & I \\
X_{0} & X_{0}
\end{array}\right]^{-1}\left[\begin{array}{l}
C_{0} \\
C_{1}
\end{array}\right]=\left[\begin{array}{cc}
I / 2 & X_{0}^{-1} / 2 \\
I / 2 & -X_{0}^{-1} / 2
\end{array}\right]\left[\begin{array}{l}
C_{0} \\
C_{1}
\end{array}\right]
$$

From here the result is concluded.

The following result yields a characterization in order to assure that the only solution of the problem (1.1) is the trivial one. In the terminology of the scalar case, this means that $\lambda$ is not an eigenvalue of the Sturm-Liouville problem (1.1).

Theorem 1. Let us consider the boundary value problem (1.1), where $\lambda \neq 0$ and $Q$ is an invertible operator such that

$$
\sigma(\lambda Q)=\{\lambda z ; z \text { belongs to } \sigma(Q)\} \subset D_{\alpha}
$$

for some $\alpha$ in $\left[0,2 \pi\left[\right.\right.$, and let $\mathbf{X}_{0}=\exp \left(\left(\log _{\alpha}(\lambda Q) / 2\right)\right.$. If the operator matrix

$$
S=\left[\begin{array}{cc}
E_{1}+E_{2} X_{0} & E_{1}-E_{2} X_{0} \\
\left(F_{1}+F_{2} X_{0}\right) \exp \left(a X_{0}\right) & \left(F_{1}-F_{2} X_{0}\right) \exp \left(-a X_{0}\right)
\end{array}\right]
$$

is invertible, then the only solution of the Problem (1.1) is the trivial $X(t)=0$ for all $t$ in $[0, a]$. If $H$ is finite-dimensional, the invertibility of $S$ is a necessary and sufficient condition in order to assure that the only solution is the trivial one.

Proof. From Lemma 2, the general solution of the operator differential equation (2.1) takes the form expressed by (4.2). If we impose that $X(t)$ given by (4.2) satisfies the boundary value conditions of (1.1), it follows that the operators $C$ and $D$ must verify the conditions

$$
\begin{gathered}
E_{1}(C+D)+E_{2}\left(X_{0} C-X_{0} D\right)=0 \\
F_{1}\left(\exp \left(a X_{0}\right) C+\exp \left(-a X_{0}\right) D\right)+F_{2}\left(\exp \left(a X_{0} C-\exp \left(-a X_{0}\right) X_{0} D\right)=0\right.
\end{gathered}
$$


or equivalently,

$$
\begin{gathered}
\left(E_{1}+E_{2} X_{0}\right) C+\left(E_{1}-E_{2} X_{0}\right) D=0 \\
\left(F_{1}+F_{2} X_{0}\right) \exp \left(a X_{0}\right) C+\left(F_{1}-F_{2} X_{0}\right) \exp \left(-a X_{0}\right) D=0 \\
S\left[\begin{array}{l}
C \\
D
\end{array}\right]=\left[\begin{array}{l}
0 \\
0
\end{array}\right] .
\end{gathered}
$$

If $S$ is invertible, the only solution of (9.2) is $C=D=0$. If $H$ is finite-dimensional, the invertibility of $S$ is also a necessary condition in order to assure that the only solution of equation (9.2) is $C=D=0$.

Remark 1. If $H$ is infinite-dimensional and the complex number 0 belongs to the compression spectrum of $S$, that is, if $S(H \oplus H)$ is not dense in $H \oplus H$, then there are nonzero operators $C$ and $D$ such that $(9.2)$ is verified and $S$ is not invertible. A lot of concrete examples are shown in the following.

Example 2. Let us consider the Problem (1.1) where $E_{2}=F_{2}=I, H$ is a complex separable Hilbert space with orthonormal basis $\left\{e_{n} ; n \geqq 1\right\}$ and let $\left\{u_{n}\right\}_{n \geqq 1}$ be a sequence of complex numbers convergent to a complex number $u$. Let us consider that $Q$ is the operator defined on $H$ by $Q\left(e_{j}\right)=0$ if $1 \leqq j \leqq k$, and $Q\left(e_{j}\right)=u_{j-k} e_{j}$, if $j>k$, and let $F_{1}=0$ and $E_{1}$ an injective unilateral weighted shift operator, defined by $E_{1}\left(e_{n}\right)=w_{n} e_{n+k}$, for some bounded sequence of complex numbers $\left\{w_{n}\right\}_{n \geqq 1}$, and for a fixed positive integer $k$. Then it follows that $X_{0}$ is given by the infinite diagonal matrix

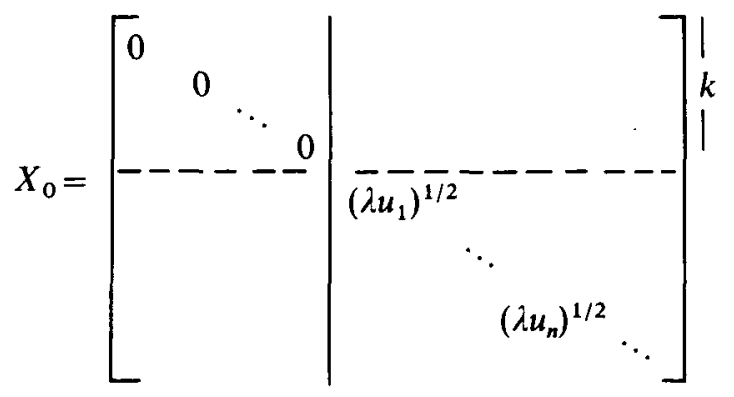

that is, $X_{0}\left(e_{j}\right)=0$ if $1 \leqq j \leqq k$, and $X_{0}\left(e_{j}\right)=\left(\lambda u_{j-k}\right)^{1 / 2} e_{j}$, for $j>k$. Let us consider $a=1$. It is easy to show that $\left(F_{1}+F_{2} X_{0}\right) \exp \left(X_{0}\right)\left(e_{j}\right)=0$ if $1 \leqq j \leqq k$, and $\left(F_{1}+F_{2} X_{0}\right) \exp \left(X_{0}\right)\left(e_{j}\right)=$ $\left(\lambda u_{j-k}\right)^{1 / 2} \exp \left(\left(\lambda u_{j-k}\right)^{1 / 2}\right)$, for $j>k$. Moreover, as the ranges $\left(E_{1}+E_{2} X_{0}\right)(H)$ and $\left(E_{1}-E_{2} X_{0}\right)(H)$ are contained in the orthogonal complementary of the subspace $M=L I N\left(\left\{e_{j} ; 1 \leqq j \leqq k\right\}\right)$. It turns out that considering $C$ and $D$ as any finite-dimensional projection with ranges contained in $M$, the condition (9.2) is satisfied.

Corollary 1. Let us consider the Problem (1.1) where $\lambda \neq 0, Q$ is an invertible operator which satisfies the condition (7.2) and $S$ is the operator defined by (8.2). Then there are 
nontrivial solutions of (1.1), if and only if

$$
0 \in \sigma_{\text {comp }}(S) \text {. }
$$

In this case the operator function defined by (4.2) is a solution of this problem, where $C$ and $D$ satisfy (9.2). If $N$ is a closed subspace of $H \oplus H$ that is orthogonal to the subspace $S(H \oplus H)$, and $N_{1}$ and $N_{2}$ are the subspaces of $H \oplus H$ defined by

$$
N_{1}=N \cap(H \oplus\{0\}), \quad N_{2}=N \cap(\{0\} \oplus H)
$$

then $C$ and $D$ can be chosen as the projections on $H$ with ranges $N_{1}$ and $N_{2}$ respectively.

Proof. It is a consequence of Theorem 1 and Remark 1.

Notice that different solutions for the Problem (1.1) can be found depending on the codimension of the subspace $S(H \oplus H)$. Lemma 1 permits us to find more concrete conditions than (10.2) in terms of data problem.

Corollary 2. Let us consider the Problem (1.1) where $\lambda \neq 0, Q$ is an invertible operator which satisfies (7.2) and $S$ is the operator defined by (8.2).

(i) If $F_{1}-F_{2} X_{0}$ is invertible, the condition (10.2) is equivalent to the condition

$$
0 \in \sigma_{\text {comp }}\left(\left(E_{1}+E_{2} X_{0}\right)-\left(E_{1}-E_{2} X_{0}\right)\left(\exp \left(a X_{0}\right)\left(F_{1}-F_{2} X_{0}\right)^{-1}\left(F_{1}+F_{2} X_{0}\right) \exp \left(a X_{0}\right) .\right.\right.
$$

(ii) If $E_{1}+E_{2} X_{0}$ is invertible, the condition (10.2) is equivalent to the condition

$$
0 \in \sigma_{\text {comp }}\left(\left(F_{1}-F_{2} X_{0}\right) \exp \left(-a X_{0}\right)-\left(F_{1}+F_{2} X_{0}\right) \exp \left(a X_{0}\right)\left(E_{1}+E_{2} X_{0}\right)^{-1}\left(E_{1}-E_{2} X_{0}\right)\right) .
$$

Proof. It is an easy consequence of the proof of Lemma 1, taking into account that if $A_{22}$ is invertible, the subspace $A(H \oplus H)$ is not dense in $H \oplus H$, if and only if $\left(A_{11}-A_{12} A_{22}^{-1} A_{21}\right)(H)$ is not dense in $H$. By application of this fact to the operator $S$, part (i) is proved. An analogous consideration related to the proof of Lemma 1 (ii), yields the proof of (ii).

Corollary 3. Let us consider the Problem (1.1) where $\lambda \neq 0, Q$ is an invertible operator which satisfies the condition (7.2) and $S$ is the operator defined by (8.2).

(i) If $F_{1}-F_{2} X_{0}$ is invertible and the condition (11.2) is satisfied, then a solution of the Problem (1.1) is given by (4.2), where $C$ is any operator that satisfies

$$
\left(\left(E_{1}+E_{2} X_{0}\right)-\left(E_{1}-E_{2} X_{0}\right) \exp \left(a X_{0}\right)\left(F_{1}-F_{2} X_{0}\right)^{-1}\left(F_{1}+F_{2} X_{0}\right) \exp \left(a X_{0}\right)\right) C=0
$$


and

$$
D=\exp \left(a X_{0}\right)\left(F_{1}-F_{2} X_{0}\right)^{-1}\left(F_{1}+F_{2} X_{0}\right) \exp \left(a X_{0}\right) C
$$

(ii) If $E_{1}+E_{2} X_{0}$ is invertible and the condition (12.2) is satisfied, then the operator function defined by (4.2) where $D$ is any operator that satisfies

$$
\left(\left(F_{1}-F_{2} X_{0}\right) \exp \left(-a X_{0}\right)-\left(F_{1}+F_{2} X_{0}\right) \exp \left(a X_{0}\right)\left(E_{1}+E_{2} X_{0}\right)^{-1}\left(E_{1}-E_{2} X_{0}\right)\right) D=0
$$

and $C=-\left(E_{1}+E_{2} X_{0}\right)^{-1}\left(E_{1}-E_{2} X_{0}\right) D$, defines a solution of (1.1).

Proof. It is a consequence of Corollary 2 and the proof of Theorem 1. Moreover, notice that from (11.2) and (12.2), the operators $C$ and $D$ can be chosen in such way that $X(t)$ given by $(4.2)$ is a non trivial solution of $(1.1)$.

The following is concerned with the study of the boundary value problem (1.1) where $\lambda=0$. In this case, the operator differential equation (2.1) takes the form $X^{(2)}(t)=0$, and it is clear that the only solution of this equation which satisfies the initial conditions $X(0)=C_{0}$ and $X^{(1)}(0)=C_{1}$, is the operator function $X(t)=C_{0}+C_{1} t$, for all $t$ in $[0, a]$.

Theorem 2. Let us consider Problem (1.1) where $\lambda=0$. Let $T$ be the operator matrix

$$
T=\left[\begin{array}{cc}
E_{1} & E_{2} \\
F_{1} & a F_{1}+F_{2}
\end{array}\right]
$$

(i) Problem (1.1) with $\lambda=0$ has only the trivial solution if and only if the subspace $T(H \oplus H)$ is dense in $H \oplus H$, that is, if $0 \notin \sigma_{\text {comp }}(T)$. In particular, if $H$ is finitedimensional, there are non trivial solutions if and only if, $T$ is invertible.

(ii) Under the hypothesis of (i), if $E_{1}$ is invertible, the operator function $X(t)=C_{0}+C_{1} t$, is a non trivial solution of (1.1), if $C_{1}$ is any operator that satisfies

$$
\left(F_{2}+F_{1}\left(a I-E_{1}^{-1} E_{2}\right)\right) C_{1}=0
$$

and $C_{0}=-E_{1}^{-1} E_{2} C_{1}$.

(iii) Under the hypothesis of (i), if $a F_{1}+F_{2}$ is invertible, the operator function $X(t)=$ $C_{0}+C_{1} t$, is a non trivial solution of (1.1) if $C_{0}$ is any nonzero operator on $H$ which satisfies

$$
\left(E_{1}-E_{2}\left(a F_{1}+F_{2}\right)^{-1} F_{1}\right) C_{0}=0
$$

and $C_{1}=-\left(a F_{1}+F_{2}\right)^{-1} F_{1} C_{0}$

Proof. If the general solution $X(t)=C_{0}+C_{1} t$, satisfies the boundary value condition 
of Problem (1.1), then it must verify the conditions

$$
\begin{gathered}
E_{1} C_{0}+E_{2} C_{1}=0 \\
F_{1}\left(C_{0}+a C_{1}\right)+F_{2} C_{1}=0
\end{gathered}
$$

or equivalently

$$
\left[\begin{array}{cc}
E_{1} & E_{2} \\
F_{1} & a F_{1}+F_{2}
\end{array}\right]\left[\begin{array}{l}
C_{0} \\
C_{1}
\end{array}\right]=\left[\begin{array}{l}
0 \\
0
\end{array}\right]
$$

thus, the existence of nonzero operators $C_{0}, C_{1}$ is equivalent to the condition $T(H \oplus H)$ not dense in $H \oplus H$. From here (i) is proved.

(ii) If $E_{1}$ is invertible, then solving in (16.2) the result is immediate.

(iii) Solving in (16.2) the result is a consequence of the invertibility of the operator $a F_{1}+F_{2}$.

Acknowledgement. This paper has been partially supported by a grant from the Conselleria de Cultura, Educació i Ciència de la Generalitat de Valencia.

\section{REFERENCES}

1. N. ARLeY and V. Borchsenius, On the theory of infinite-systems of differential equations and their application to the theory of stochastic processes and the perturbation theory of quantum mechanics, Acta Math. 76 (1944), 261-322.

2. H. Bart, M. A. Kaashoek and L. Lerer, Review of Matrix Polynomials, by I. Gohberg, P. Lancaster and L. Rodman, Linear Algebra Appl. 64 (1985), 267-272.

3. S. K. Berberian, Lectures on Functional Analysis and Operator Theory (Springer-Verlag, 1974).

4. E. A. Coddington and N. Levinson, Theory of Ordinary Differential Equations (MacGrawHill, 1955).

5. N. Dunford and J. Schwartz, Linear Operators I (Interscience, 1957).

6. I. Gohberg, P. Lancaster and L. Rodman, Matrix Polynomials (Academic Press, 1982).

7. E. L. INCE, Ordinary Differential Equations (Dover, 1927).

8. L. JódaR, Boundary value problems for second order operator differential equations, Linear Algebra and Appl., to appear.

9. L. Jódar, Boundary problems for Riccati and Lyapunov equations, Proc. Edinburgh Math. Soc. 29 (1986), 15-21.

10. J. G. Kemeny, J. L. Snell and A. W. Knapp, Denumerable Markov Chains (Van Nostrand, Princeton).

11. S. G. Krein, Linear differential equations in Banach spaces, Trans. Math. Monographs 29 (1971). 
12. P. A. MisnaevskiI, On the Green's function and the asymptotic behavior of eigenvalues of Sturm-Liouville operator problem, Soviet Math. Dokl. 13 (1972), 465-469.

13. P. A. MisnaevsKII, On the spectral theory for the Sturm-Liouville equation with operator coefficient, Math. U.S.S.R. Izv. 10 (1976), 145-180.

14. V. A. Milhailec, The solvability and completeness of the system of eigenfunctions and associated functions of nonselfadjoint boundary value problems for a Sturm-Liouville operator equation, Soviet Math. Dokl. 15 (1974), 1327-1330.

15. H. H. Murtazin, On the point spectrum of a class of differential operator, Soviet Math. Dokl. 15 (1974), 1812-1814.

16. H. H. MurTazin, On nonisolated points of the spectrum of eliptic operators, Math. U.S.S.R. Izv. 10 (1976), 393-411.

17. M. N. Oguztorelli, On infinite systems of differential equations occurring in the degradations of polymers, Utilitas Math. 1 (1972), 141-155.

18. L. Rodman, On factorization of operator polynomials and analytic operator functions, Rocky Mountain J. Math., to appear.

19. A. L. SHIELDS, Weighted Shift Operators and Analytic Function Theory (Math. Surveys No. 13, C. Pearcy, Ed., Amer. Math. Soc., 1974).

20. S. Steinberg, Infinite systems of ordinary differential equations with unbounded coefficients and moment problems, J. Math. Anal. Appl. 41 (1973), 685-694.

Department of Applied Mathematics

Polytechnical University of Valencia

P.O. Box 22. 012

VALENCIA

SPAIN 